\title{
SERIAL VERSUS CONCURRENT MULTITASKING: FROM LAB TO LIFE
}

\author{
Paul W. Burgess \\ Institute of Cognitive Neuroscience \\ University College London \\ 17 Queen Square, London WC1N3AR, U.K. \\ p.burgess@ucl.ac.uk
}

Chapter to appear in J. Fawcett, E. F. Risko \& A. Kingstone (Eds.), The Handbook of Attention. MIT Press. 


\section{Introduction}

"Multitasking" is a very recent term. Its first use is widely attributed to Witt and Ward (1965) in an IBM technical manual describing a computer operating system. However, its application in describing human behaviour is much more recent, and a key issue is emerging. This is that "multitasking" is being used to describe (at least) two situations that might be distinct from each other in psychological terms.

The first situation is where people are actively trying to do two or more tasks at the same time. Examples might be driving while making a phone call, or reading while simultaneously watching a film. Many recent examples are also given in the literature in relation to "media multitasking", i.e. attempting to attend simultaneously to stimuli from multiple media sources (e.g. Ophir et al, 2009; see also Chapter 23 in the current volume).

The second is where people have a "to-do" list of jobs or tasks in their minds, and are trying to optimise their behaviour in order to achieve the best outcome in relation to them. An example might be cooking a complex meal while also preparing the house for guests, and dealing with the demands of children.

When people in everyday life describe themselves as "multitasking", they may be using the term to refer to either of these situations almost interchangeably. And indeed, organised everyday life when seen over substantial time periods can often include examples of both, sometimes with the first form embedded within the second in complex ways. In both situations, there is an intention to perform more than one task, typically within a certain time-frame. But in the second situation there is no attempt to perform more than one task at the same time. Following Salvucci and Taatgen (2008), the term "concurrent multitasking" will be used to describe the first situation. For the second situation, this chapter will introduce the term "serial multitasking". This paper will argue that there are significant differences between these two forms of multitasking, and that recent evidence from cognitive neuroscience may give some clues as to fruitful ways of pursuing these differences. 


\section{Historical Context}

Although the term "multitasking" is a recent invention, it would be wrong to conclude that research relevant to human multitasking was not carried out before the invention of this term. In other words, it is the term "multitasking" that is new, rather than research into the corresponding mental phenomena.

Until recently, dealing with the demands of multiple tasks was known as "multiple-task performance". This was, in practice, most often the study of dual-task type paradigms where more than two tasks were used. In other words, it was the study of concurrent multitasking. The first book (to my knowledge) on that topic, was Diane Damos' "Multipletask Performance", published less than 25 years ago (Damos, 1991). In that book, Brookings and Damos (1991) point out that research into the nascent field of individual differences in multiple-task performance dates back more than 100 years, to e.g. the dual-tasking studies of Binet (1890) and Sharp (1899). They made the point that previously the most obviously relevant constructs or behavioural descriptions under examination were neither "multitasking" nor "multiple-task performance", but were theoretical abilities described variously as "power of distribution of attention", "division of attention" and "timesharing". This latter term is especially important, since it is prima facie a behavioural description rather than a construct one, so may well either refer to concurrent or serial multitasking. Ackerman, Schneider and Wickens (1984) provided a definition: "time-sharing ability refers to the ability to perform multiple tasks in combination" (p. 71). Thus in practice, "timesharing" was synonymous with the term "concurrent multitasking" as it is defined today. It is the study of this behaviour (concurrent multitasking) which has been the overwhelming focus for studies and theorising in the cognate sciences, in particular experimental psychology, and more recently, computational modelling (see e.g. Keiras et al, 2000). There have been various interesting demonstrations from these experiments that may have relevance for understanding everyday life performance. For instance, Schumacher et al (2001) have argued that after only moderate practice, some people can manage almost 
perfect time-sharing between two basic choice reaction tasks, and Ophir et al (2009) demonstrated that heavy "media multitaskers" in everyday life performed more poorly on a test of task-switching ability, and argued that this is likely due to a reduced ability to filter out interference from irrelevant material.

Historically, however, a recurring theme for the study of multitasking has concerned less the determinants of performance than whether there is a specific ability that is tapped by multitasking situations beyond that required for performance of the individual tasks themselves. From the earliest days this was bound up with notions of "intelligence". One of the earliest views was expounded by McQueen (1917), which is a publication of his DSc thesis from 1915 at the University of London, U.K. The topic of the research was attributed to Charles Spearman, who was the laboratory director, and provided guidance for McQueen's research until rejoining the Army on the outbreak of war. This link with Spearman may have been influential in the initial hypothesis for the research. McQueen wrote: "We certainly believe...that the assumption is without justification that a single test...affords a reliable measure of an individual's "power of distribution" of attention...We shall show...evidence indicating that there is no such general power as a power to distribute the attention successfully" (p. 4).

Spearman was of course the principal proponent of the notion of the existence in the mind of a general intelligence factor (or " $g$ "). The main evidence for Spearman's hypothesis was the repeated finding of "positive manifold" (i.e. more correlations that were positive than would be expected by chance) in correlation matrices of large numbers of people performing various disparate tasks. Perhaps it would have been awkward - or at least a complication - for the " $g$ " hypothesis if there was an ability (multitasking) that existed outside the specialist skills required for the individual subtasks. To have "specific factors" playing such a super-ordinate role in behaviour might challenge the purity of the notion of " $g$ ". So from the earliest days there may have been scepticism about the idea that multitasking involved an ability that was distinct from general intelligence. We will return to the issue of multitasking and intelligence below, because cognitive neuroscience has recently offered some startling new evidence that speaks to it, suggesting that this scepticism was not warranted. 
Despite this early scepticism, the search for a specific "multitasking ability" continued. It was hampered however by the lack of information processing models that specified what operations such an ability would support, and the relation of this ability to others within the cognitive system (e.g. Ackerman et al, 1984). Ackerman et al (1984) presented the view quite clearly. They contend that when considering multitasking data from any one individual, there are specific sets of variance attributable to individual differences in ability to perform the subtasks, but also another quite separate source of variance that represents the persons' time-sharing (aka multitasking) ability. They proposed that, as an example, that where two subtasks are being considered, approximately $25 \%$ of the score might be attributable to multitasking ability (with $37.5 \%$ attributable to each subtasks, assuming zero error variance). So, for Ackerman et al, multitasking definitely represents a special ability above and beyond those required to perform the subtasks themselves. As mentioned above, this chapter will outline modern cognitive neuroscience evidence that supports such a view, and it even suggests basic information about the neural substrates of multitasking ability.

The confidence of Ackerman's view contrasted with the main bulk of thinking from the 1970s and 1980s. Brookings and Damos (1991) reviewed the evidence for a separate multitasking ability emerging from both factor analytic and non-factor analytic studies during that period, usually involving dual-task type paradigms. With notable exceptions (e.g. Sverko, Jerneic and Kulenovic, 1983), these studies failed to find evidence of such an ability. However, Brookings and Damos pointed out that most if not all of the principal studies had major limitations in the methodological design and/or analysis that would have limited the chances of discovery of one (i.e. an ability that would be used to deal with many different types of multitasking situation, as opposed to abilities that might be involved in dealing with only a limited subset of them) should one exist.

There were nevertheless some interesting investigations. For instance, one way in which a general multitasking ability might be demonstrated would be if training in multitasking could be shown to generalise to other situations. Damos and Wickens (1980) examined performance on two task combinations to try to discover evidence that multitasking (or in 
their terminology, "timesharing") skills are learned with practice and can transfer between task combinations. The first task combination consisted of a short-term memory task and a classification task; the second consisted of two identical one-dimensional compensatory tracking tasks, requiring participants to keep a moving circle centred within a horizontal track by using a control stick. One task was controlled by each hand. The experiment used three groups of participants. The first received dual-task training on both task combinations. The second received single-task training on the first task combination, and dual-task training on the second combination (the tracking). The third was given dual-task training on the second (tracking) combination only (i.e. no single-task training). By contrasting these groups, Damos and Wickens (1980) were able to examine the degree to which performance on the dual-task tracking task benefited from prior dual-task exposure. They found that transfer of learning was positive: earlier dual-task training did seem to develop a generalizable dualtask skill. However, as pointed out by Lintern and Wickens (1991), the amount of transfer of timesharing adaptation from one situation to another was modest relative to the skill learning demonstrated on the individual subtasks. Moreover, they contend, "most timesharing skills may be specific to a given task combination and may not be of the generic type" (p. 136). This begs the question of the definition of a multitasking ability.

In these ways, the issue of whether there are specific multitasking skills has historically been contentious. One suggestion presented here is that the source of this contention is that there may be different abilities that underpin behaviour in different multitasking situations. Thus it might have been more fruitful to have sought "multitasking abilities" (e.g. independent of " $g$ ") rather than look for evidence of a single common one. Thus a wider consideration of the range of multitasking situations than has been considered historically, and especially in relation to multitasking demands that present themselves in everyday life, may yield more definitive experimental results.

\section{State-of-the-Art Review}

It should be clear from the foregoing historical context that the emerging field of "multitasking" has several issues at the heart of it. The first of these is that there is little 
formal distinction concerning whether the term refers to a behaviour, a construct, or a situation. In other words, does it refer to the act of doing several things at once - in which case are examples like walking whilst talking examples of multitasking? Or does it refer to a theoretical ability, in the same way as e.g. the term "working memory"? Or does it refer to a situation that is presented to a participant (e.g. three novel tasks are presented to a person with the instruction that they should attempt to do them all "at the same time")? At various times in the past, terms have been used that seem to relate very strongly to the more recent term "multitasking" that might have been thought of as constituting any one of these.

In this treatise, "multitasking" will be used in reference to the third kind of definition. In other words, that it defines a set of features of a situation that are presented to an individual. Ideally we then should be able to hone these features into a cardinal set that would distinguish multitasking situations or paradigms from any other (e.g. a dual-task paradigm, or a working memory paradigm etc.). If this definition of the term is adopted, it leaves open the matter of whether people who are multitasking are actually "doing several tasks at once". This is useful, since it may side-step the traditional concerns of dual- or multiple-tasking (e.g. the nature of the psychological refractory period, or the switch cost in task-switching paradigms; Monsell and Driver, 2000). It is not that these issues are not important - far from it - but more that there is an opportunity with the new term to reorient the concerns of the area towards the everyday, at least for those who see that as desirable. In other words, to create experimental paradigms as starting points for investigation that deliberately model the demands of "real-world", or "everyday" situations. Such a starting point may help offset the danger of becoming "phenomenon-driven" (Newell, 1973; Monsell and Driver, 2000) and thus encourage the paradigms used for research to be more closely aligned to the everyday situations for which experimental paradigms are usually intended to be a model. As Kieras et al (2000) put it, "future research must use a wide variety of empirical procedures to investigate multitask performance. This investigation should extend beyond basic laboratory paradigms like the task-switching and PRP procedures, which...come nowhere near to engaging the whole host of executive mental processes that people presumably have. Rather, to explore these processes...overlapping-task procedures with complex realistic tasks and unpredictable 
stimulus-response event sequences will be needed". As we will see below, it is exactly these sorts of procedures that have proved a starting point for one particular line of investigation of multitasking within cognitive neuroscience.

\section{Serial vs. concurrent multitasking}

Interestingly an "everyday situation" is taken as the reference situation in the theoretical paper of Salvucci and Taatgen (2008). This paper aims to form a model of the demands made by concurrent multitasking. But these researchers do not take as a starting point a typical multiple-tasks type experimental paradigm. Instead, the cardinal example of a situation requiring multitasking that they describe is cooking in a busy kitchen. They imagine a cook trying to fulfil multiple food orders simultaneously. While some tasks can be carried out in parallel (e.g. baking fish in an oven while pasta boils on a stove), in many situations this cannot happen because either the same resource (e.g. oven) needs to be used for two separate tasks, or the cook is physically unable to perform two distinct complex actions at the same time. Salvucci and Taatgen (2008) point out that in this situation the cook needs to delay performing one action while another is carried out (p. 103). This everyday example illustrates well how multitasking in everyday life is often not a matter of a person attempting to do two tasks at the same time, as is asked of a participant in a typical experimental concurrent multitasking paradigms (e.g. those historically called dual- or multiple-task paradigms). Instead, much of the behaviour is more closely aligned to the definition of serial multitasking presented here. Thus we may have the beginning of a discontinuity between the demands of everyday life and those of experimental practice.

Of course, I provide absolutely no evidence whatsoever for this contention (i.e. that much of dealing with several tasks in everyday life takes the form of serial rather than concurrent multitasking). But the motivation for it is to try to capture the essence of what people in "everyday life" (i.e. outside academia) mean by the term, in the hope that such a endeavour might guard against "phenomena-driven" experimental work - or at least that the phenomena under examination are those observed in the "real-world" rather than only the lab. 
So let us consider what an "everyday" definition of a multitasking situation might look like. For this argument, let us leave aside the issue for the moment of concurrent vs. serial multitasking, and seek the broadest level of definition that encompasses all forms. We cannot start and end with the characteristic of "doing several tasks at once", since people routinely perform two tasks at the same time: It depends what you call a "task". So for instance, someone who is walking whilst holding a conversation could be argued to be performing two tasks simultaneously (i.e. transport to a destination, and having a discussion), and therefore "dual-tasking". If one then adds the behaviour where that person is also opening an umbrella, then prima facie this might be "multitasking". However, if one stopped a thousand people on a busy London street and asked them to give an example of a typical situation that requires "multitasking", few would probably give an example this mundane. Instead, running through the examples that one would get would probably be the following characteristics:

Characteristic 1: There are several tasks that are desired to be performed, and for some reason they cannot be done in pure sequence, i.e. task 1 , task 2 , task 3 , etc. Instead, adequate performance will either: (a) require interleaving of responses, thoughts or actions in relation to the different tasks, e.g. task 1 , task 2, task 1 , task 3, task 2 etc and/or (b) require the person to be trying to do more than one task "at the same time". It is the "trying" that is the important feature here, since it is a moot point as to whether many complex mental tasks can actually be performed "at the same time" when examined at the millisecond level. In this way (b) may actually be (a) when observed scientifically.

Characteristic 2: There is novelty in either the combination or order of the subtasks being attempted. Highly overlearned sequences of co-ordinated behaviours, such as occurs for instance when an experienced driver is driving (e.g. checking the rear-view mirror while switching on an indicator) is unlikely to be described as "multitasking". However introduce a novel requirement (or even better, several) into the sequence (e.g. ask the driver to count how many times he touches the brake medal in a 5 minute period) and most people would probably consider that as an example of some form of multitasking. 
Characteristic 3: This might refer to the distinctness of the tasks and therefore the level of interference between them. So, talking to two people in a three-way conversation is unlikely to be seen as a strong example of "multitasking" behaviour. But using two telephones to conduct two different conversations with different people at the same time probably would be, despite the fact that here there are also three people talking, and only one may be talking at a time, just as before. So captured in this "everyday knowledge" is perhaps a notion of holding some distinct stream of thought in mind (in this case the currently unattended conversation) whilst indulging in another stream of thought in relation to another goal or activity (the other conversation).

Of course, there are many observations one might make about these characteristics. For instance, different multitasking situations will differ in the degree to which they show these characteristics. And the characteristics themselves are not easy to measure. Novelty, in particular, presents all manner of measurement and methodological problems (Burgess, 1997).

Another aspect is the intent of the participants (see Characteristic 1) as much as the situation that is presented to them. Indeed, one of the most common ways in which multitasking situations differ from each other is in the intent of the participants, and the way they conceive of the parameters of the task. The priority they assign is particularly important. In dual-task paradigms or multiple-task ones, the participant is typically instructed to try to perform both tasks at once. This does not mean however that the two tasks necessarily are given equal priority. A good example is Gopher (1993), who assigned equal priorities to two subtasks (visuo-manual tracking and a choice-reaction time (CRT) task) in one group of participants who were then given a series of dual-task-type practice sessions. In another group, the priorities for the subtasks were varied: sometimes it was the tracking task that was given priority, sometimes the CRT, and sometimes they were assigned equal priority. Gopher found that variable-priority training led to better performance on the two subtasks than fixed-priority training. This characteristic - that the participant is trying to perform two or more tasks at the same time (whether this is psychologically possible or not) is prima facie a feature of concurrent multitasking paradigms. 
However, as mentioned above, many everyday situations are not like the situation presented by Gopher. Instead, they often require serial multitasking. This is where, for practical or other reasons, only one task can be attempted at a time. The participants is not trying to do multiple tasks at the same time, but they are trying to get several tasks done within a certain time period, and for one reason or another they are not able to do each task from start to finish in strict sequence.

While experimental psychology has concerned itself principally with studying concurrent multitasking situations, human neuropsychology (and to a lesser extent, cognitive neuroscience more broadly) has largely concerned itself with the study of serial multitasking situations (with a few notable exceptions, e.g. Baddeley et al, 1997). One major difference between the two fields is that neuropsychology is driven to a greater degree by observation of people (in this case, patients) in everyday life. And it is serial multitasking situations where many patients with neurological damage to a certain part of the frontal lobes show a particular difficulty. These neuropsychological studies have clear implications for creating information processing models of the differing demands of these two multitasking situations, and so the data is highly relevant to experimental psychology and cognitive science, even though it is not often recognised.

\section{Everyday multitasking deficits in neurological patients: lessons for building information processing models}

What have we learned from neuropsychological studies about the nature of the organisation of the processes involved in serial multitasking? Well, the most obvious lesson from neurological patients is that deficits in serial multitasking ability can be seen in the context of intact intellect, as well as intact episodic memory, language, visuo-motor, visuoperceptual skills, as well as intact ability on most commonly used problem-solving tasks. This was first demonstrated empirically by Shallice and Burgess (1991). Thus neuropsychology provides strong evidence for a special (i.e. independent of " $g$ ") multitasking facility of the kind that was sought by experimental psychologists for many decades, with rather equivocal results. 
Shallice and Burgess (1991) described three people who had all suffered frontal lobe damage following traumatic brain injury. All three had no significant impairment on formal tests of perception, language and intelligence and two of them performed well on a variety of traditional tests of novel problem-solving. But they all demonstrated severe problems in everyday life, especially with situations that required serial multitasking. Shallice and Burgess (1991) invented two new tests of multitasking to quantify these problems. The first is called the "Multiple Errands Test", and is a real-life multitasking test typically carried out in shopping precinct. Participants are pre-trained on a set of rules (e.g. no shop should be entered other than to buy something; spend as little money as possible), and then are asked to undertake a number of tasks while following them. The tasks vary in terms of complexity (e.g. buy a small brown loaf vs. discover the exchange rate of a particular foreign currency yesterday), and there are a number of "hidden" problems in the tasks that have to be appreciated and the possible course of action evaluated. For instance, the participants are required to write and send a postcard, but (a) they are given no pen; (b) they are instructed not to use anything not bought on the street to help them, and (c) they are also told that need to spend as little money as possible. In this way, the task is very "open-ended" or "illstructured", meaning that there are many possible courses of action, and it is up to the individual to determine for themselves which one they will choose, and to a certain extent, what constitutes acceptable performance.

Clearly, a task as complex as the MET taps many different abilities that relate to novel problem-solving, prioritization and so forth on top of pure multitasking ability. So in order to measure the multitasking component in a more controlled way, Shallice and Burgess invented a test called the "Six Element Test". This required subjects to swap between 3 simple subtasks, each divided into two sections, within 15 minutes, whilst following some arbitrary rules (e.g. "you cannot do part A of a subtask followed immediately by part B of the same subtask). They were told that they should attempt at least some items of all subtasks. It was up to the participant to decide when to switch tasks since no signal was given, and although a clock was present, it was covered, so that checking it has to be a deliberate action of lifting the cover to check it. 15 minutes was not long enough to complete all the subtasks (or even all of one subtask), so the participants needed to 
remember to switch from one task to another before reaching completion, so that they have at least attempted all subtasks. The Six Element Test therefore has a strong component of voluntary time-based task switching (see Burgess et al, 2009, 2011; GonenYaacovi and Burgess, 2012 for review).

All three cases reported by Shallice and Burgess all performed both the MET and Six Element tests at a level below the 5th percentile level compared with age- and IQ-matched controls. On the MET the subjects made several different types of error. For instance, they found themselves having to go into the same shop several times, when they could have bought all they needed at one visit. They also forgot to carry out tasks that they intended to do, and often broke the tasks rules. There were also many examples of curious social behavior and interactions (e.g. offering sexual favours in lieu of payment). These kinds of social issues are a regular occurrence in this type of patient and it may potentially be instructive as to the basis of e.g. social attention (see Chapter 15 in the current volume, for an outline of the mechanics of social attention). Shallice and Burgess (1991) termed this kind of behavioural disorganization in the context of preserved intellect and other cognitive functions the "Strategy Application Disorder". This term was chosen as to be reasonably neutral about range of difficulties that these patients might show upon further investigation. But one defining feature seemed to be impairment in situations requiring serial multitasking.

On the specificity of the multitasking impairment in neurological patients.

The demonstration that serial multitasking impairments can be seen in the context of e.g. intact IQ is a strong indication for a specific ability that underpins at least some forms of multitasking. However, one might argue that the types of tasks used above tap more than just multitasking abilities. For instance, they may require planning, and "monitoring" (i.e. keeping a record of where you are in a goal list), and remembering of the task rules. So Burgess et al (2000) used the group lesion study methodology to investigate whether impairments in these sorts of stages of performance (e.g. planning) were a root cause of multitasking impairments. Sixty acute neurological patients and 60 age- and IQ-matched 
healthy controls were administered a multitasking test similar to the Six Element Test. Before participants began the test, their ability to learn the task rules (by both spontaneous and cued recall) was measured. They were then asked how they intended to do the test, and a measure of the complexity and appropriateness of their plans was gained. This enabled us to look at whether their failures could be due to poor planning. The participants then performed the task itself and by comparing what they did with what they had planned to do, a measure of "plan following" was made. Multitasking performance itself was measured as the number of task switches minus the number of rule breaks. After these stages were finished, subjects were asked to recollect their own actions by describing in detail what they had done. Finally, delayed memory for the task rules was examined.

This procedure enabled us to demonstrate the specificity of the deficit in patients who fail the test in the context of good planning, comprehension and (retrospective) memory abilities. Critically, we found that patients with left hemisphere rostral PFC lesions (i.e. the most anterior regions of prefrontal cortex, variously also referred to as frontopolar cortex or Brodmann's area 10), when compared with patients with lesions elsewhere, showed a significant multitasking impairment despite no significant impairment on any variable except the one reflecting multitasking performance. In other words, despite being able to learn the task rules, form a plan, remember their actions, and say what they should have done, they nevertheless did not do what they said that they intended to do.

Notably, perhaps, we have recently found that patients with rostral PFC lesions show problems with certain tasks which require time perception (Volle et al, 2011). How this relates to multitasking problems we do not currently know. But it may turn out to be instructive (e.g., see Chapter 3 in the current volume, for discussion of temporal attention).

Whatever the precise cause, the link between serial multitasking deficits and damage to rostral PFC has been replicated using the group lesion method first by Dreher et al (2008), and then John Duncan's group in Cambridge, UK (Roca et al, 2010, 2011). Roca et al's (2010) study is particularly noteworthy in the current context in that it showed that multitasking deficits were still seen even after the scores were adjusted for change in a specific measure of "fluid intelligence" (Cattell's Culture Fair test). So after 20 years of research into serial 
multitasking deficits in humans, we seem to have reached as good an agreement as scientists usually can that serial multitasking is a behavior that makes demands upon a resource that is independent from "IQ", and that there is an area within rostral PFC that seems at least in part to support it.

\section{Integration}

\section{The cognitive demands of serial multitasking}

If serial multitasking performance is not strongly related to $I Q$, or a range of other cognitive/problem-solving abilities, then what might be the processes that support it? This section will consider some possibilities.

Clearly, the situations presented to the participants when they are asked to do the MET and SET are very different from e.g. a typical dual-task or multiple-task paradigm used in the experimental psychology literature. Specifically, the characteristics of the MET and SET have been described by Burgess and Wu (2012) and are detailed in Table 1. Characteristic 6 is not a necessary feature and may not occur in most experimental situations (like the Six Element Test when given clinically), but is an added and important determinant of performance in typical everyday life.

\section{TABLE 1 HERE}

The findings of a specific link between serial multitasking deficits and rostral PFC damage invites (although it does not provide) a first-pass constraining of the possible candidate mental processes that supports the behavior to those that have been associated with rostral PFC function. So what are they?

Rostral PFC structures have been found to support, at least in part, a wide range of mental abilities. These include analogical reasoning, maintenance and execution of delayed intentions (aka prospective memory), source and context memory, mentalising, mind- 
wandering and other stimulus-independent mental phenomena, time perception, humour judgment, and task initiation speed (Burgess, Gonen-Yaacovi and Volle, 2012; Burgess and Wu, 2013; Gilbert et al, 2007, 2012). One theory of the role of rostral PFC in cognition is that it underpins an attentional "gateway" that allows one to control the degree to which one is engaging in stimulus-independent thought (e.g. an inner mental dialogue) versus attending to external stimuli. This theory is known as the "gateway hypothesis", and the theoretical construct underpinning this mental ability is known as "XN attending control" (Burgess et al, 2005, 2006, 2007).

Why should the information processing associated with these types of behaviours be particularly associated with performance in serial multitasking situations, especially everyday-type ones? One possibility is that while it is the case that the kinds of mental phenomena associated with rostral PFC function may have little relation to performance of the kinds of highly constrained and very structured tasks that are typically administered to participants in a brain scanner, or in an experimental psychology study, this may not be true of very open-ended serial multitasking tasks like those that have the characteristics outlined in Table 1. In typical fMRI experimental tasks, for instance, it is ideal to have a large number of repeated trials or responses (often presented in pseudo-random order to avoid artifact), and it is nearly always very clear to the participant what exactly is required of them, and whether they are succeeding or not. However this is NOT typical of many or maybe even most tasks in everyday life, at least outside the classroom or a very repetitive job. In more "realistic" experimental tasks, or open-ended or "ill-structured" everyday life situations, metacognitive introspections about e.g. how well one is doing, or what is expected of one, may well one highly helpful to task performance. In this way there may be consistent patterns of perceptual decoupling occurring (for more information about these kinds of phenomena please see Chapter 10 in the current volume). So recent research into the functions of rostral PFC is suggesting that there are many cognitive abilities that are specifically designed to help individuals to be competent in everyday life, but are not at all well-captured in many experimental situations.

In my lab, we have been examining the plausibility of the hypothesis that $\mathrm{XN}$ attending control (see above) is a central feature to the performance of tasks which involve activation 
of a delayed intention, which is a central feature of many serial multitasking situations (see Characteristic 4 in Table 1). Benoit et al. (2012) designed an fMRI paradigm which parametrically crossed $\mathrm{XN}$ control with a task involving maintaining and executing a delayed intention. We found that there was considerable overlap in the brain regions that showed BOLD signal change during the $\mathrm{XN}$ control manipulation and those involved in conditions involving a delayed intention (see also Burgess et al, 2011; Volle et al, 2011). These results are consistent with the hypothesis that being able to effect good control over the direction of one's attending is an important determinant of being able to schedule and interleave a series of tasks within a certain time-frame (i.e. serial multitasking).

By contrast, perhaps concurrent multitasking makes fewer demands upon processes involved in dealing with delayed intentions. This might be because the "goal list" or "goal instructions" for each task are being consciously rehearsed, or are, to use the frame work of John Duncan, integrated into a single "task model" (Duncan et al, 2008). If this was the case, then one might expect in neuroimaging experiments to see a different pattern of activation during concurrent multitasking compared with serial multitasking.

Etienne Koechlin and his colleagues have examined this situation directly. Instead of "multitasking", Koechlin has used the term "cognitive branching" to describe "a domaingeneral core function that enables the brain to contingently put on hold one behavioural option/task in order to perform another one" (Koechlin 2013, p. 285). This provides a rather good description of the situation being investigated by researchers interested in multitasking (especially serial multitasking), and indeed, in later work (e.g. Dreher et al, 2008) the construct "cognitive branching" was re-described as "multitasking". The task that Koechlin introduced was ingenious. There were four conditions, and in each, individual letters (appearing in either upper or lower-case) from the word "tablet" were presented one-at-a-time to participants. In the first, control condition, participants were asked to judge whether two successively presented letters were in immediate succession in the word 'tablet' (only upper-case letters were presented). In the second condition ("delay condition"), participants were also asked to decide if two successively presented upper-case letters occurred in immediate succession in the word "tablet". But this time they were asked to ignore lower case letters. This meant that there was a delay period for upper-case letters. 
The third condition was a dual-task condition. Here, participants also had to decide if successively presented letters (paying attention to both upper can case letters) were in immediate succession in the word "tablet". They also had to decide if each first letter indicating a case change was the letter $\mathrm{T}$ (or $\mathrm{t}$ ). The fourth condition was the multitasking condition. Here, they essentially had to do both of the previous tasks. So for successive upper case letters, or for successive lower case letters, participants decided whether the current letter followed immediately the previously presented letter in the word "TABLET" (or 'tablet"). And they also had to decide whether each first letter signifying a case change was the letter $\mathrm{T}$ (or $\mathrm{t})$.

Koechlin et al (1999) found bilateral BOLD signal increases during the dual task condition in the posterior dorsolateral prefrontal cortex BA (Brodmann's area) 9, the middle frontal gyrus, close to the pre-central gyrus (BA 8), and the lateral lateral inferior parietal lobule (BA 40). There were no specific brain regions identified in the delayed condition. However, in the multitasking condition there were blood-oxygen level dependent (BOLD) signal increases found bilaterally in dorsal aspects of the rostral prefrontal cortex (i.e. the frontal pole, or BA 10). BOLD signal increase in these regions only occurred in the multitasking condition, and was similar in the other conditions, including the control condition. This meant that the rostral prefrontal cortex activations could not be explained by differences in "task difficulty" as indexed by task performance, since that would have been reflected in a gradual increase from the control to the delayed task dual-task and branching conditions. One of the key aspects of Koechlin's study was that it gives evidence that dual-task performance and serial multitasking are not identical in terms of the neural architecture that supports them. It is tempting to conclude that perhaps this is the result of concurrent performance of more than one task, and serial performance of them, being psychologically different.

\section{Future directions}

Research into the cognitive and brain basis of multitasking behaviour has, in scientific terms, only just started. There are early signs that the criticisms that were made of dual-tasking 
research thirty years or so ago (e.g. lack of theoretical underpinning; paradigm-bound research) may not also be made of this emerging field. There are useful theoretical models emerging (e.g. Salvucci \& Taatgen, 2008), partly because we now have methods for theory testing that were only incipient back then (e.g. computational modelling). And we now have demonstration of neurological patients with circumscribed lesions that have caused circumscribed multitasking problems, in effect answering a question that is nearly 100 years old: whether there is a specific multitasking ability beyond that which is required for performance of the subtasks themselves. If patients with rostral prefrontal cortex (brain area 10) damage can be absolutely unimpaired at the subtasks themselves but show catastrophic impairment in organising their performance when many tasks are put together, then prima facie this is strong evidence that putting several subtasks together makes demands beyond that of the individual subtasks together. Moreover, there is confirmatory evidence in terms of localisation from neuroimaging - again, a new method that did not start to exert itself until the mid-1990s.

One main current focus in the cognitive neuroscience of multitasking is trying to understand how the brain supports the ability to remember to carry out an intended act at a future time (i.e. prospective memory), since this seems highly relevant to understanding serial multitasking. Dual-task or concurrent multitasking type paradigms seem to make demands upon different parts of the brain than serial multitasking. Since those regions are often also closely associated with "working-memory" type paradigms, one possible explanation consistent with these results is that concurrent multitasking ability may be relatively more related to active rehearsal or maintenance of a goal set, whereas serial multitasking may stress relatively more those process relating to delayed intentions. Accordingly, for serial multitasking, a key concern for behavioural study becomes that of "intention cost", first demonstrated behaviourally (as far as I am aware) by Burgess et al (2003; Figure 1, p. 910). This is the cost of performance upon the current task you are performing caused by maintaining a delayed intention, in this case, to do the next scheduled task. There are echoes here of Witt and Ward (1965) who said "half-completed tasks, in a multitasking operation, tie up the system facilities and prevent other tasks from proceeding" (p. 58). How this relates to e.g. the aspect of novelty of the subtasks and the combinations of them is 
however not known, and this may be a promising avenue of enquiry for experimental psychologists.

An attempt at a simple behavioural analysis of the characteristics of serial multitasking has been attempted here. But it is preliminary, and the defining characteristics of concurrent multitasking situations are as yet not well established either. Clearly, we would all agree that "multitasking situations" are where someone is dealing with multiple tasks or demands. But what might we say beyond that, in terms of instructions to participants, or their aims, or the nature of the subtasks? How much does one have to alter a subtask (and in what way) before it can be considered a "different task"? Are well-rehearsed combination of actions and behaviours (like walking whilst talking) examples of multitasking, and if not, why not? It seems that the issue of the novelty of the combination of actions or tasks is likely key to our definition but there is relatively little work in that area as yet.

A fascinating possibility for understanding the basis of multitasking is presented by Messinger et al (2009), who claim to have discovered "multitasking neurons" in the prefrontal cortex of primates. In their experiment, monkeys were required to attend to a visual stimulus at one location while remembering a second place. Most neurons specialized in either attentional or mnemonic processing. But about one-third of the cells responded to features of both, and they termed these "multitasking neurons". They argue that these neurons afford an advantage for situations where two or more demands are made simultaneously. They do this by having stronger tuning than more specialised cells, and expressing preferred directions for attention and for working memory (they may even oppose one another). In this way, Messinger et al argue that pairs of multitasking neurons can represent cognitive parameters more efficiently than pairs that include even a single specialized cell. This view may have received further momentum by the recent finding from Takeuchi et al (2013) who gave healthy young adults four weeks of concurrent multitasking training, and examined the brain changes that occurred over that period (in regional grey matter volume, and also in resting functional connectivity). They found that concurrent multitasking training was accompanied by increased regional grey matter volume in three subregions of the prefrontal cortex (left lateral rostral PFC; dorsolateral PFC, and the left inferior frontal junction) as well as a set of regions outside the frontal lobes (left posterior 
parietal cortex; left temporal and lateral occipital regions). They also found decreased resting-state connectivity between the right dorsolateral prefrontal cortex and the ventral anterior cingulate cortex. Takeuchi et al (2013) argue that these results may suggest that there are some neural changes that are not tightly restricted to specific task conditions. There are resonances here perhaps with John Duncan's (2001) adaptive coding theory of the properties of prefrontal cortex. The notion that frontal neurons may have a less tightly specialised functional capacity than neurons elsewhere in the cortex is an attractive one. However, at least for rostral PFC, there is considerable evidence now for a large degree of functional specialisation within this large brain region (Burgess et al, 2007; Burgess, GonenYaacovi and Volle, 2012; Burgess and Wu, 2013), and it does not seem implausible that there may be neurons that are highly involved with e.g. maintaining an intention over a delay period. Whether this sort of function can be linked very specifically to a particular behaviour once we understand the operations they perform at the information processing level remains to be discovered.

But at this very early stage of investigation into multitasking, many of the basic questions have not even yet been asked, never mind answered. For instance, there is surprisingly little integration between the literature on task switching (see Chapter 7 in the current volume for a review) and that of multitasking, despite its obvious relevance. A good place to start seeking these questions will be to look at the behaviours in everyday life that we label as multitasking, and ask what demands they make, and then create experimental analogues of them. This is already occurring in some fields (e.g. Hancock et al, 2003). This chapter argues that this approach in the neuropsychology of complex behaviours has been fruitful. It contrasts with a "construct" (rather than behavioural) led approach that e.g. first posits a multitasking construct (independent of a particular behaviour) and then builds a paradigm to test that theory, with little regard to whether the resulting task is anything like situations that are encountered in everyday life either now or in human history. One danger of the latter approach is that we may end up studying situations that are so divorced from those that the cognitive system will have developed to deal with (in an evolutionary sense) that we may be examining curiosities of the system rather than mechanisms fundamental to mainstream behaviour (Burgess et al, 2006). 



\section{KEY POINTS}

- Concurrent multitasking is where a person is attempting to do several tasks at the same time (e.g. talking on a mobile phone while shopping).

- Serial multitasking is where people are interleaving several tasks but can only do one at a time. This interleaving often occurs because one task cannot be finished before another must start (e.g. when cooking a meal).

- Serial and concurrent multitasking may make different cognitive demands.

- Serial and concurrent multitasking may be supported, at least in part, by different brain structures, or at least by operation of the same brain regions to different degrees.

- Serial multitasking ability seems to a surprising degree to be unrelated to general intellectual ability, as measured by many current IQ tests. It is not yet well established whether concurrent multitasking is similarly unrelated.

\section{OUTSTANDING ISSUES}

- In what ways do open-ended multitasking tests (i.e. where the participants decide for themselves what to do and when) make different cognitive demands from ones where the optimal course is decided by the experimenter?

- In what ways are well-practiced multitasking situations different from novel ones, at information-processing and neural levels?

- What are the respective roles of dorsolateral and rostral prefrontal cortex in multitasking?

- Do serial and concurrent multitasking abilities change over the lifespan in the same way?

- Theoretical models of all forms of multitasking are urgently needed. 


\section{REFERENCES}

Ackerman, P. L., Schneider, W. and Wickens, C. D. (1984) Deciding the existence of timesharing ability: A combined methodological and theoretical approach. Human Factors 26, 71-82.

Baddeley, A., Della Sala, S., Papagno, C. and Spinnler, H. (1997) Dual-task performance in dysexecutive and nondysexecutive patients with a frontal lesion. Neuropsychology 11(2), 187-194.

Benoit, R.G., Gilbert, S.J., Frith, C.D., and Burgess, P.W. (2012). Rostral prefrontal cortex and the focus of attention in prospective memory. Cerebral Cortex 22 (8), 1876-1886.

Binet, A. (1890) La concurrence des etats psychologiques. Revue Philosophique de la France et L'Etranger, 29, 138-155.

Burgess, P. W. (2000) Real-world multitasking from a cognitive neuroscience perspective. In: S. Monsell and J. Driver (Eds.) Control of Cognitive Processes: Attention and Performance XVIII (pp. 465-472). Cambridge, MA: MIT Press.

Burgess, P. W. (1997) Theory and methodology in executive function research. In: P. Rabbitt (Ed.) Theory and Methodology of Frontal and Executive Function (pp. 81-116). Hove, U.K.: Psychology Press.

Burgess, P. W., Alderman, N., Forbes, C., Costello, A., Coates, L. M-A., Dawson, D. R., Anderson, N. D., Gilbert, S. J., Dumontheil, I. and Channon, S. (2006) The case for the development and use of "ecologically valid" measures of executive function in experimental and clinical neuropsychology. Journal of the International Neuropsychological Society 12, 116.

Burgess, P. W., Alderman, N., Volle, E., Benoit, R. G. \& Gilbert, S. J. (2009) Mesulam's frontal lobe mystery re-examined. Restorative Neurology and Neuroscience 27(5):493-506.

Burgess, P. W., Dumontheil, I., \& Gilbert, S. J. (2007). The gateway hypothesis of rostral prefrontal cortex (area 10) function. Trends in Cognitive Sciences, 11, 290-298. 
Burgess, P. W., Gilbert, S. J., Okuda, J. and Simons, J.S. (2006) Rostral Prefrontal Brain Regions (Area 10): A Gateway between Inner Thought and the External World? In: W. Prinz \& N. Sebanz (Eds.), Disorders of Volition (pp. 373-396). Cambridge, MA: MIT Press.

Burgess, P.W., Gonen-Yaacovi G., and Volle E. (2012) Rostral prefrontal cortex: What neuroimaging can learn from human neuropsychology. In Brian Levine and Fergus I. M. Craik (Eds.) Mind and the Frontal Lobes (pp. 47-92). New York: Oxford University Press.

Burgess, P.W., Gonen-Yaacovi, G, and Volle E. (2011). Functional neuroimaging studies of prospective memory: What have we learnt so far? Neuropsychologia 49(8): 2246-2257.

Burgess, P. W., Simons, J. S., Dumontheil, I., and Gilbert, S. J. (2005) The Gateway Hypothesis of Rostral PFC Function. In: J. Duncan, L. Phillips \& P. McLeod (Eds.) Measuring the Mind: Speed, Control and Age (pp. 215-246). Oxford University Press.

Burgess, P. W., Veitch, E., Costello, A. and Shallice, T. (2000) The cognitive and neuroanatomical correlates of multitasking. Neuropsychologia $38,848-863$.

Burgess, P. W. \& Wu, H-C. (2013) Rostral prefrontal cortex (Brodmann area 10): metacognition in the brain. Chapter 31 in: Principles of frontal lobe function, $2^{\text {nd }}$ edition (Editors: Donald T. Stuss \& Robert T. Knight) pp. 524-534. New York: OUP.

Brookings, J.B. and Damos, D L. (1991) Individual differences in multiple-task performance. In D. L. Damos Ed.), Multiple-Task Performance, pp. 363-386. London: Taylor and Francis. Damos, D. L. (1991) Multiple-Task Performance. London: Taylor and Francis.

Damos, D. L. and Wickens, C. D. (1980) The identification and transfer of timesharing skills. Acta Psychologica, 46 (1), 15-39.

Dreher, J-C., Koechlin, E., Tierney, M., and Grafman, J. (2008) Damage to the fronto-polar cortex is associated with impaired multitasking. PLoS ONE 3(9): e3227. doi:10.1371/journal.pone.0003227

Duncan, J. (2001). An adaptive coding model of neural function in prefrontal cortex. Nature reviews Neuroscience 2, 820-829. DOI: 10.1038/35097575 
Duncan, J., Parr, A., Woolgar, A., Thompson, R., Bright, P., Cox, S., Bishop, S., and NimmoSmith, I. (2008) Goal neglect and Spearman's g: competing parts of a complex task. Journal of Experimental Psychology General 137(1), 131-48.

Gilbert, S.J., Bird, G., Frith, C.D., \& Burgess, P.W. (2012). Does "task difficulty" explain "taskinduced deactivation"? Frontiers in Cognition, April 2012 Vol. 3, Article 125.

Gilbert, S. J., Dumontheil, I., Simons, J. S., Frith, C. D., \& Burgess, P. W. (2007) Comment on "Wandering minds: the default network and stimulus-independent thought". Science, 317(5834), 43.

Gonen-Yaacovi, G. and Burgess, P. W. (2012) Prospective memory: the future for future intentions. Psychologica Belgica 173 52/2-3, 173-204.

Gopher, D. (1993). The skill of attention control: Acquisition and execution of attention strategies. In D. Meyer \& S. Kornblum (Eds.). Attention and Performance XIV: Synergies in Experimental Psychology, Artificial Intelligence, and Cognitive Neuroscience - A Silver Jubilee Volume (pp. 299-322). Cambridge, MA: MIT Press.

Hancock, P.A., Lesch, M., Simmons, L. (2003). The distraction effects of phone use during a crucial driving maneuver. Accident Analysis and Prevention 35, 501-514.

Lintern, G. and Wickens, C. D. (1991) Issues for acquisition and transfer of timesharing and dual-task skills. In: D. L. Damos (Ed.) Multiple-Task Performance. London, UK: Taylor \& Francis. ISBN 0-85066-757-7.

Kieras, D. E., Meyer, D. E., Ballas, J. A. and Lauber, E. J. (2000) Modern computational perspectives on executive mental processes and cognitive control: Where to from here? In S. Monsell and J. Driver (Eds.), Control of Cognitive Processes: Attention and Performance XVIII (pp. 681-712). Cambridge, MA: MIT Press.

Koechlin, E., Basso, G., Pietrini, P., Panzer, S. and Grafman, J. (1999). The role of the anterior prefrontal cortex in human cognition. Nature, 399(6732), 148-151. 
Koechlin, E. (2013). Motivation, control, and human prefrontal executive function. In D.T. Stuss and R.T. Knight (Eds.) Principles of Frontal Lobe Function, $\left(2^{\text {nd }}\right.$ Edition). New York: Oxfrod University Press. ISBN 978-0-19-983775-5.

McQueen, E. N. (1917) The distribution of attention. British Journal of Psychology 11 (Monograph Supplements, 5).

Monsell, S. and Driver, J. (2000) Banishing the control homunculus. In S. Monsell and J. Driver (Eds.), Control of Cognitive Processes: Attention and Performance XVIII (pp. 3-32) . Cambridge, MA: MIT Press.

Newell, A. (1973) You can't play twenty questions with nature and win. In W. A. Chase (Ed.), Visual Information Processing (pp. 283-308). New York: Academic Press.

Ophir, E., Nass, C., and Wagner, A. D. (2009). Cognitive control in media multitaskers. PNAS 106( 37), 15583-15587. doi: 10.1073/pnas.0903620106

Roca, M., Parr, A., Thompson, R., Woolgar, A., Torralva, T., Antoun, N., Manes, F., and Duncan, J. (2010). Executive function and fluid intelligence after frontal lobe lesions. Brain. 133(1), 234-247.

Roca, M., Torralva, T., Gleichgerrcht, E., Woolgard, A., Thompson, R., Duncan, J. , Manes, F. (2011) The role of Area 10 (BA10) in human multitasking and in social cognition: A lesion study. Neuropsychologia 49, 3525-3531.

Salvucci, D. D. and Taatgen, N. A. (2008) Threaded cognition: An integrated theory of concurrent multitasking. Psychological Review 115 (1), 101-130.

Schumacher, E.H. Seymour, T. L., Lauber, E. J., Kieras, D. E., Meyer, D. E. Glass, J. M., and Fencsik, D. E. (2001) 'Virtually Perfect Time Sharing in Dual-Task Performance: Uncorking the Central Cognitive Bottleneck', Psychological Science 12 (2): 101-8. 
Shallice, T. and Burgess, P. W. (1991) Deficits in strategy application following frontal lobe damage in man. Brain 114, 727-741.

Sharp, S. (1899) Individual psychology: A study in psychological method. The American Journal of Psychology 10, 329-91.

Sverko, B., Jerneic, Z., and Kulenovic, A. (1983) A contribution to the investigation of timesharing ability. Ergonomics, 26, 151-160.

Takeuchi, H., Taki, Y., Nouchi, R., Hashizume, H., Sekiguchi, A., Kotozaki, Y., Nakagawa, S., Miyauchi, .CM., Sassa, Y., Kawashima, R. (2013). Effects of multitasking-training on gray matter structure and resting state neural mechanisms. Human Brain Mapping 12, Dec 17. doi: 10.1002/hbm.22427. [Epub ahead of print]

Volle, E., Gonen-Yaacovi, G., de Lacy Costello, A., Gilbert, S.J., Burgess P.W. (2011). The role of rostral prefrontal cortex in prospective memory: A voxel-based lesion study. Neuropsychologia 49(8): 2185-98.

Witt, B. I. \& Ward, L. (1965). IBM operating system/360 concepts and facilities. IBM Systems Reference Library File Number S360-36, form C28-6535-0. 
TABLE 1

\section{Characteristics of typical serial multitasking situations in everyday life according to Burgess (2000).}

1. A number of discrete and different tasks have to be completed.

2. Performance on these tasks needs to be dovetailed in order to be time-effective.

3. Due to either cognitive or physical constraints, only one task can be performed at any one time.

4. The appropriate stages for returns to task are not signaled directly by the situation.

5. There is no moment-by-moment performance feedback of the sort that participants in many laboratory experiments will receive. Typically, failures are not signaled at the time they occur.

6. Unforeseen interruptions, sometimes of high priority will occasionally occur, and things will not always go as planned.

7. Tasks usually differ in terms of priority, difficulty and the length of time they will occupy.

8. People decide for themselves what constitutes adequate performance. 